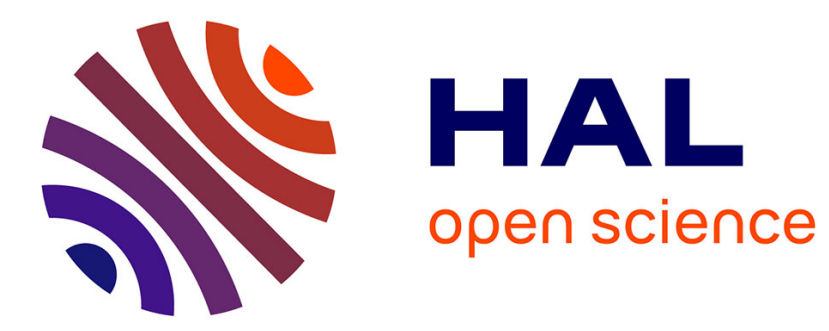

\title{
Assessing the Functional Feasibility of Variability-Intensive Data Flow-Oriented Systems
}

Sami Lazreg, Philippe Collet, Sébastien Mosser

\section{To cite this version:}

Sami Lazreg, Philippe Collet, Sébastien Mosser. Assessing the Functional Feasibility of VariabilityIntensive Data Flow-Oriented Systems. Symposium on Applied Computing, Apr 2018, Pau, France. 10.1145/3167132.3167354. hal-01660057

\section{HAL Id: hal-01660057 https://hal.science/hal-01660057}

Submitted on 9 Dec 2017

HAL is a multi-disciplinary open access archive for the deposit and dissemination of scientific research documents, whether they are published or not. The documents may come from teaching and research institutions in France or abroad, or from public or private research centers.
L'archive ouverte pluridisciplinaire HAL, est destinée au dépôt et à la diffusion de documents scientifiques de niveau recherche, publiés ou non, émanant des établissements d'enseignement et de recherche français ou étrangers, des laboratoires publics ou privés. 


\section{Assessing the Functional Feasibility of Variability-Intensive Data Flow-Oriented Systems}

\author{
Sami Lazreg \\ Visteon Electronics and Université \\ Côte d'Azur, CNRS, I3S, France \\ slazreg@visteon.com
}

\author{
Philippe Collet \\ Université Côte d'Azur, CNRS, I3S, \\ France \\ collet@i3s.unice.fr
}

\author{
Sébastien Mosser \\ Université Côte d'Azur, CNRS, I3S, \\ France \\ mosser@i3s.unice.fr
}

\begin{abstract}
Data-flow oriented embedded systems, such as automotive systems used to render HMI (e.g., instrument clusters, infotainments), are increasingly built from highly variable specifications while targeting different constrained hardware platforms configurable in a finegrained way. These variabilities at two different levels lead to a huge number of possible embedded system solutions, which feasibility is extremely complex and tedious to predetermine. In this paper, we propose a tooled approach that capture high level specifications as variable dataflows, and targeted platforms as variable component models. Dataflows can then be mapped onto platforms to express a specification of such variability-intensive systems. The proposed tool support transforms this specification into structural and behavioral variability models and reuses automated reasoning techniques to explore and assess the feasibility of all variants in a single run. We also report on the application of the proposed approach to an industrial case study of automotive instrument cluster.
\end{abstract}

\section{CCS CONCEPTS}

- General and reference $\rightarrow$ Design; Validation; • Computer systems organization $\rightarrow$ Embedded systems; $\bullet$ Software and its engineering $\rightarrow$ Software product lines; • Theory of computation $\rightarrow$ Verification by model checking;

\section{KEYWORDS}

Embedded system design engineering; variability modeling; feature model; behavioral product lines model checking.

\section{ACM Reference Format:}

Sami Lazreg, Philippe Collet, and Sébastien Mosser. 2018. Assessing the Functional Feasibility of Variability-Intensive Data Flow-Oriented Systems. In SAC 2018: SAC 2018: Symposium on Applied Computing, April 9-13, 2018, Pau, France. ACM, New York, NY, USA, 10 pages. https://doi.org/10.1145/ 3167132.3167354

\section{INTRODUCTION}

Validating embedded systems design at early stages of development is of fundamental importance in industry. Ideally embedded system design should be modeled from high-level specifications, and then

Publication rights licensed to ACM. ACM acknowledges that this contribution was authored or co-authored by an employee, contractor or affiliate of a national government. As such, the Government retains a nonexclusive, royalty-free right to publish or reproduce this article, or to allow others to do so, for Government purposes only.

SAC 2018, April 9-13, 2018, Pau, France

( 2018 Copyright held by the owner/author(s). Publication rights licensed to Association for Computing Machinery.

ACM ISBN 978-1-4503-5191-1/18/04 .\$15.00

https://doi.org/10.1145/3167132.3167354 assess against possible implementations. Data-flow oriented embedded systems, such as automotive systems used to render HMI (e.g., instrument clusters, infotainments) are typically built from highly variable specifications. They are composed of a data-flow driving and feeding graphical processors to provide efficient and high-quality graphic rendering at a lower cost, while targeted hardware platforms are composed of heterogeneous and constrained hardware components. The variability is then two-fold, with multiple graphic data-flow variants that can meet functional requirements, and diverse targeted hardware platform, which are highly configurable in a fine-grained way. These dimensions of variability dreadfully increase the size of the design space of these embedded systems (i.e., the number of possible embedded system implementation designs), making the feasibility assessment of these systems extremely tedious and complex.

Generally, design spaces of variable systems and protocols are assessed through variability-aware model checking on variable transition-based systems [1,9]. However these approaches are not capable of automatically map the variable data-flow specifications on configurable platform descriptions to apply their modelchecking techniques. Consequently, this would imply to manually infer, model and assess embedded system design spaces from high level specifications, making this activity extremely tedious, time consuming, and error-prone.

Facing this issue, we determine three challenges to be tackled: (i) capturing and modeling from high-level specifications, structure, behavior and variability of these embedded systems (e.g., data-flow and platform alternatives, data sizes, memory capacities, graphic pipelines), (ii) inferring automatically all possible embedded system design implementations from specification models and, (iii) exploring and assessing the feasibility of all system implementations w.r.t. the predefined structural, behavioral and variability constraints. Current approaches $[15,16,23]$ assess functional feasibility of constrained data-flow-oriented embedded systems, but do not capture nor manage variability at both levels. Some Ad hoc techniques are trying to handle either platform variability (as reconfigurable architectures [21, 22][19]) or functional variability (as multiple scenarios [20, 25] or multi-modes systems [18, 26]). On the other hand, approaches tackling both kinds of variability [12] are focusing on optimal platform selections to implement multiple functional variants at a lower cost, but they do not manage structural and behavioral properties (e.g. data sizes, memory capacities, graphic pipelines).

In this paper we propose an approach that extends these researches by supporting a complete modeling and assessment of structural, behavioral and variability properties of the targeted embedded systems by combining embedded system design engineering 
$[15,16,23]$ and software product line engineering techniques $[1,9]$. The proposed framework is model driven and i) capture high level variable data-flow and platform specifications following principles of separation of concern, ii) maps variable data-flow requirements into a description of the targeted variable hardware platform, so to infer the embedded system design space (i.e. all system implementations), iii) transforms the design space into a behavioral product line to reuse automated reasoning techniques (i.e. SAT solving, variability-aware model checking) to explore and assess the functional feasibility of all system design implementations in a single run. The framework also allows to remove invalid designs from the design space by constraining it.

The remainder of the paper is organized as follows. Section 2 introduces the context and motivations illustrated by a running example. Section 3 presents the proposed framework, detailing each model and step. In Section 4 we report on first experimental results obtained from the application of the framework implementation on a real industrial use case in automotive systems. Section 5 concludes the paper.

\section{MOTIVATIONS}

Requirement gathering and validation of this research work have been realized in the context of an industrial collaboration with Visteon Electronics, a world class leader in automotive systems (e.g. instruments clusters, infotainment, connected vehicles). In the following we introduce one of the company case studies, extract a running example, determine requirements from them and discuss related work.

\subsection{Case study}

The case study is focusing on functional validation of some instrument clusters. By applying various data-flow image processing effects, such as blending, warping and scaling, an instrument cluster system improves driver experience with useful and high quality 2D/3D Human-Machine Interface (HMI). The embedded hardware platforms used to develop these systems are more and more highly configurable, but constrained in terms of architecture and capacities. Furthermore, multiple graphic data-flows variants can meet the client HMI requirements, but they also depend on the platform architectures and capacities. We consider this case study as representative of variability-intensive data-flow oriented systems. Different forms of variability, from high-level data-flows to lowlevel platforms lead to a huge number of possible system solutions, which feasibility is extremely complex and tedious to predetermine early in the development process.

\subsection{Running Example}

We now introduce a running example of a simplified instrument cluster. The data-flow on Fig. 1 represents different image flow processing that meet the HMI functional requirements. Images are processed by graphical tasks: image $d_{2}$ has two different possible resolutions (e.g. 800x480, 480x320) and will be processed by task $C$. Image $d_{1}$ can be either processed by task $A$ or task $B$.

On the hardware side (cf. Fig. 2), the platform provides image processing capabilities through non programmable pipeline processors of DCU (Display Controller Unit) or GPU (Graphic Processing Unit)

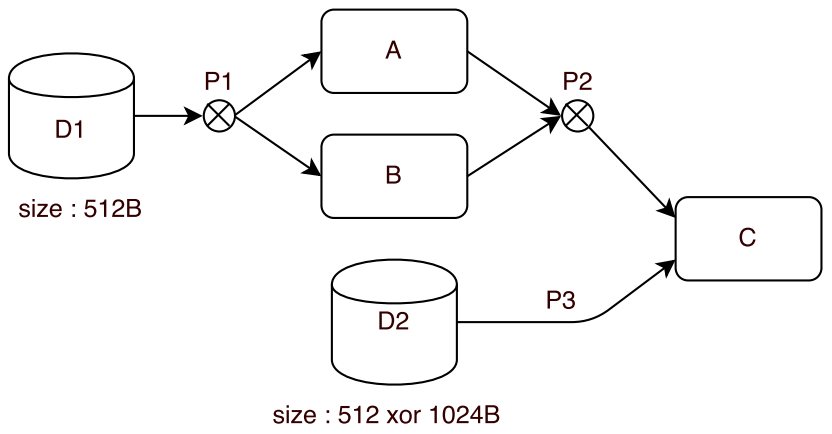

Figure 1: Functional specification

type, as well as data storage functionalities through RAM (Random Access Memory) and ROM (Read Only Memory). RAM and GPU are optional in the actual hardware products, so a system implementation may contain or not these components. Among variabilities in platforms, one can write data into and/or read data from RAM memory while only reading data is possible from ROM. Moreover, memory storage have limited and possibly variable (e.g. RAM) capacity. Contrary to a DCU, which renders directly processed images into display, a GPU needs to store its processing result into RAM. Graphical hardware processors are often designed as a multi-step pipeline, composed of several hardware implemented processing steps, and processor internal fifo memory buffers transferring data from one step to another. In our example, while a GPU can apply $A$ followed by $B$ processing on images in a single pass, a DCU can apply $A$, then followed by $C$.

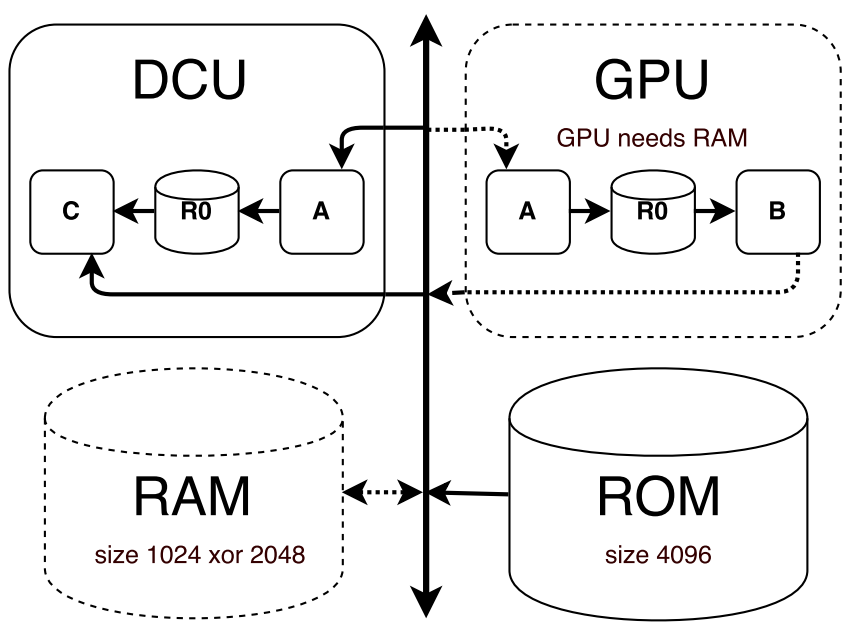

Figure 2: Platform specification

Images and processing could be, respectively, stored and processed by multiple components, while images can be stored on RAM or on ROM: task $A$ could be processed by both GPU and DCU, but a data-flow variant containing $B$ task can only be implemented on a system containing a GPU. Finally, data mapping on memory are constrained in terms of storage capacity. 
Consequently, to be valid, a system implementation has to fulfill (i) structural constraints such as not violating maximum memory capacities, (ii) behavioral constraints such as using correctly processor pipelines and memories, and (iii) variability constraints such as component dependency and exclusion. In our example, the application data-flow has 4 variants while the Platform exposes 3 architecture variants. Even with this simplified case, this leads to 178 possible implementations, in which 58 satisfy constraints and could be developed by engineers. ${ }^{1}$

\subsection{Related Work}

In the context of our work, engineers must be assisted to assess the functional feasibility of the different potential embedded system solutions, with means to capture both the high-level functional requirements and the specifications of targeted variable platforms. Ideally, a solution should be able to capture structural, behavioral and variability properties of both functional and platform specifications at a fine-grained level, so to use these input models to automatically infer all possible embedded system implementation designs and assess the resulting consistent design space.

In the software product line engineering, lots of approaches[1, 9] are capable to model variable transition-based systems such as safety-critical systems or protocols, and to validate, through variability-aware model-checking, temporal properties and behavioral aspects. However, given high-level data-flows and platform specifications, these approaches are not capable of automatically map data-flows on platforms in order to infer and assess the resulting design space. Assessing the different mapping manually is not feasible in practice, as the activity would be tedious and error-prone.

In embedded system design engineering, most of the approaches capture high-level application and platform specifications, and map an application on hardware platforms in order to find, by design space exploration, an optimized system implementation for a single functional specification on a single platform [15, 23]. Consequently, they do not capture nor manage variability at the application level and hardware platform variability is limited to component capacities (e.g. memory and bus size, processor frequency). Some other approaches try to handle some limited variability in functional specifications (e.g. optional task, alternative tasks, variable data) (as multiple scenarios [20,25] or multi-modes systems [18, 26]), but they do not manage platform variability. Some others try to handle some limited variability in platforms (e.g. optional resource, resource dependency, memories sizes) with reconfigurable architectures [21, 22][19]). To the best of our knowledge, none of these approaches handle variability in both application and platform sides so to assess the feasibility of our class of problem.

Interestingly, the recent approach of Graf et. al. $[13,14]$ manages some variability in both platform and functional specifications. On the platform variability side, resource components can be selected or not, while optional and mutually exclusive task groups are managed on the functional part. However, the approach is handling a coarse-grain form of requirements and cannot capture some of our

${ }^{1}$ Finding the best solution among the remaining correct solutions is also an important problem, but out of the scope of this paper. specifications (e.g.data and memory sizes, as well as platform aspects such as processor pipelines or fifo buffers). Additionally, only structural validation of the system implementations is supported. Behavioral properties (e.g. data sizes, memory capacities, graphic pipelines) and behavioral constraints (e.g., absence of deadlock, reachability, liveness, safety etc.), which are fundamental in our case, cannot be checked.

\section{PROPOSED FRAMEWORK}

\subsection{Overview}

The proposed approach follows a model driven decomposition, based on the well-known robust Y-Chart pattern [2, 16], which separates application and platform into different concerns. This allows modular specification and reasoning about the different parts of the specified embedded systems. Given high-level variable dataflow and platform inputs that notably captures the variability of functional and platform specifications, the framework will i) map all implementation of each data-flow variants on each platform configuration, ii) generate a Featured Transition System (FTS) [9] from the system design space model, i.e. representing system implementations (cf. Fig. 3). This model consists in an extended form of automaton product line, which is then iii) checked in one run by a variability-aware model checker.

As shown on Fig. 3, our framework consists of three main models and two processes. We give here an overview while the following sections will detail and illustrate these elements.

Variable applications: a functional expert is in charge of capturing the functional requirements (cf.fig. 1) of the embedded system through an extended data-flow (cf. sec. 3.2). This model contains the classic structure and behavior of the data-flow (data, task, data-path, etc.), but also captures the variability in both structural properties (e.g., data size) and behavioral properties (e.g., alternative flows).

Variable platforms: on this side, a platform expert is in charge of expressing the platform specification (cf. fig. 2) as a templated component based system (cf. sec. 3.3). This model contains a set of components connected with each others. Similarly to the application one, the platform model also captures the variability of the defined components.

Variability-aware mapping process: The mapping algorithm (cf. sec. 3.4) consumes the application and platform input models previously defined and generates the Variability-Intensive Design Space, i.e. representing system implementations. It is made of two steps: (i) it finds for each task data and data-path (cf. fig. 1) all the possible mappings on appropriate platform processors and storage (cf. fig. 2); basically this is done by matching the task names with the names of the hardware functions of processors; data-paths are mapped on reachable memory of appropriate processor hardware functions; (ii) as the design space contains all mapping possibilities, the algorithm prunes unfeasible mappings w.r.t. structural and variability constraints.

Design space as a behavioral product line: From the system design space model, a Behavioral Product Line representing all system implementations is generated (cf. sec. 3.5). This product line is represented as a featured automaton, so that we can reuse and adapt techniques that rely on variability-aware model-checking to validate the inferred systems. The basic idea is to transform a variable 


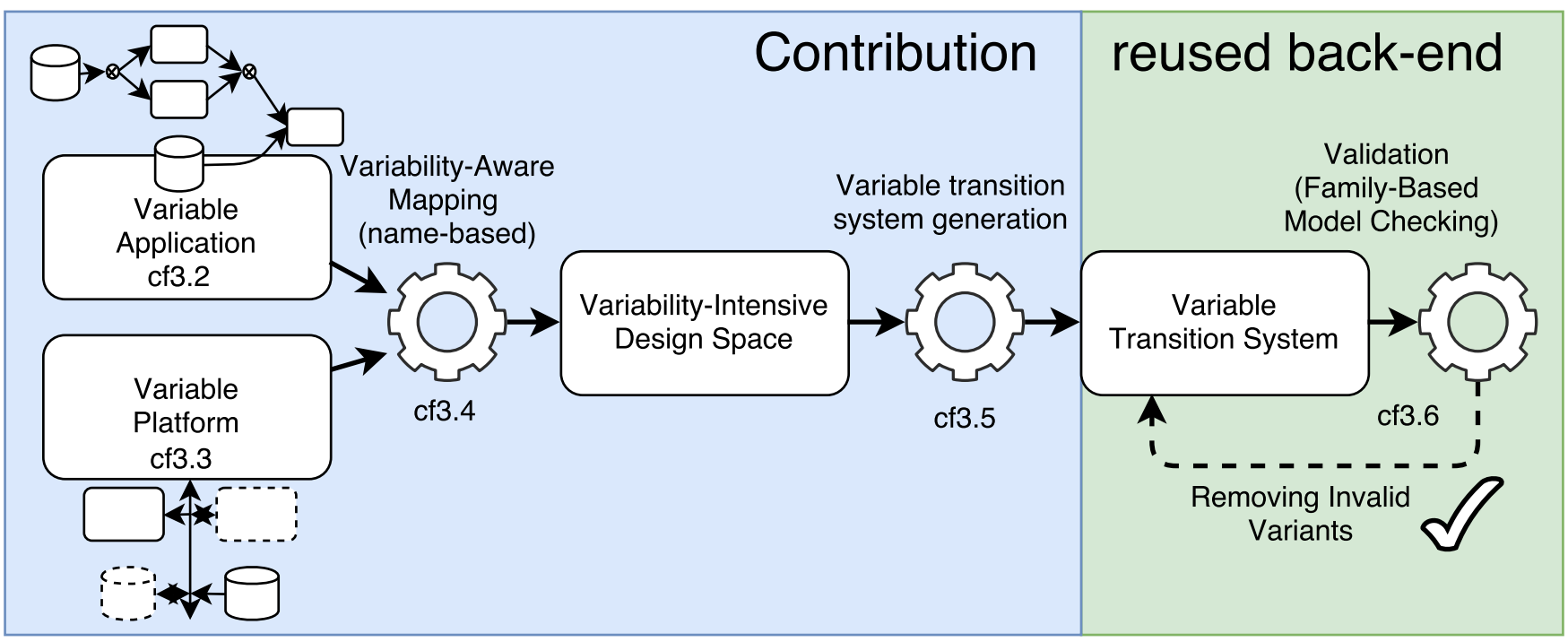

Figure 3: Framework Overview

data-flow, a variable platform, and mappings to a data-flow automaton using, through a mapping automaton, a platform automaton to execute it. Valid executions of the application automaton should then respect generated properties representing end state reachability to ensure that the execution is correctly scheduled and executed onto the platform automaton.

Validation process: The validation process reuses automated reasoning techniques to assess structural and behavioral functional feasibility of the system variants represented by the behavioral product line (cf. sec. 3.6). The model checking is going to determine classic properties, such as safety, absence of deadlock, and our state reachability generated property, on all variants in one run. As a result, the validation solves and extracts valid variants respecting all structural, behavioral and variability constraints.

\subsection{Applications as Variable Data-Flows}

In our approach, a functional expert captures structure, behavior (data, task, data-path, etc.) and variability aspects (data size, alternative flows, etc.) of the functional requirements of the embedded system through an extended data-flow model. The extensions concern variability and data aspects of functional requirements, and in the following, we propose a formal data-flow model to do so.

Definition 1. A variable data-flow graph is a tuple $V D G=(T, D$, Path $, E, \zeta)$ where:

- $T$ is a set of tasks,

- $D$ is a set of source data, and, $\zeta: D \rightarrow\left\{s_{0}, \ldots, s_{i} \in \mathbb{N}^{*}\right\}$ returns a set of alternative sizes of data,

$|\zeta(d \in D)|= \begin{cases}>1, & \text { ifd has a variable size } \\ 1, & \text { ifd has not a variable size }\end{cases}$

- Path is a set of data-paths by which producer and consumer (i.e. tasks and data) are connected.

- $E \subseteq(T \cup D) \times$ Path $\times T$ is the set of edges representing flows processing between producers and consumers.
The set of connected, input data-paths to a task I( $t)$, output data-paths from a task or data $O(v)$ are denoted by:

$I(t):\{p \in P a t h \mid(x, p, t) \in E\}$,

$O(v \in T \cup D):\{p \in P a t h \mid(v, p, x) \in E\}$.

Similarly $I(p)$, input tasks to a output data-paths, and $O(p)$, output tasks from a input data-paths, are denoted by:

$I(p):\{\operatorname{prod} \in T \cup D \mid(\operatorname{prod}, p, x) \in E\}$,

$O(p):\{t \in T \mid(x, p, t) \in E\}$.

$|I(p)|+|O(p)|=\left\{\begin{array}{ll}>2, & \text { if } p \text { has alternative flows } \\ 2, & \text { if } p \text { has not flow variability }\end{array}\right.$,

A variable data-flow represents multiple data-flow variants. To implicitly represent all these variants in a single model, we follow the same approach as in variable workflows from [13], allowing data-paths to have multiple input and output tasks connected.

A data-path can be connected to multiple alternative, input tasks if $|I(p)|>1$, and output tasks if $|O(p)|>1$. But, If $|I(p)|=1 \wedge$ $|O(p)|=1$, the data-path is connected to only one input and output task (i.e the data-path has no flow variability).

As data can have alternative sizes, we introduce the function $\zeta$ which returns the set of alternative sizes $S=\zeta(d)$, each datum has at least one size and if $|\zeta(d)|>1$ the size of $d$ is variable.

If the data-flow has no flow variability, $\forall p \in P a t h,|I(p)|+$ $|O(p)|=2$, and no data variability, $\forall d \in D,|\zeta(d)|=1$, the data-flow is not variable.

The functional specification of the case study $V D G_{u c}$ is then represented as

$$
\begin{aligned}
& \left(T_{u c}=\{a, b, c\}, D_{u c}=\left\{d_{1}, d_{2}\right\}, P a t h_{u c}=\left\{p_{1}, p_{2}, p_{3}\right\}\right. \\
& \left.E_{u c}=\left\{\left(d_{1}, p_{1}, a\right),\left(a, p_{2}, c\right),\left(d_{1}, p_{1}, b\right),\left(b, p_{2}, c\right),\left(d_{2}, p_{3}, c\right)\right\}\right) \\
& \text { with, } \\
& \zeta\left(d_{1}\right)=512, \zeta\left(d_{2}\right)=\{512,1024\} \\
& I\left(p_{1}\right)=d_{1}, I\left(p_{2}\right)=\{a, b\}, I\left(p_{3}\right)=d_{2} \\
& O\left(p_{1}\right)=\{a, b\}, O\left(p_{2}\right)=c, O\left(p_{3}\right)=c \\
& I(a)=I(b)=p_{1}, I(c)=\left\{p_{2}, p_{3}\right\} \\
& O(a)=O(b)=p_{2}, O(c)=\emptyset, O\left(d_{1}\right)=p_{1}, O\left(d_{2}\right)=p_{3}
\end{aligned}
$$




\subsection{Platforms as Variable Resource Graphs}

A variable platform specification is represented by a templated component based system (multi-pass processors, streaming processor, read-only memory, read-write memory, write-only memory, firstin-first-out buffers etc) where platform can have optional resource components and variability constraints on resources (dependency, incompatibility, etc.). To capture variability aspects of a platform specification, we propose a formal architecture model defined as follows.

Definition 2. A variable resource graph is a tuple $V R G=\left(\right.$ Proc, $\left.S, C_{S}, \xi, \theta, \phi_{\text {requires }}, \phi_{\text {excludes }}\right)$ where:

- Proc $=\left(F, B, C_{b} \subseteq(F \times B) \times(B \times F)\right)$ is a processor composed of a set $F$ of possible functions, $B$ is a set of processor internal first-in-first-out buffers and $C_{b}$ the connections between the different functions and buffers representing the processor pipeline.

- $S$ is a set of memory storage, and $\xi: S \rightarrow\left\{c_{0}, \ldots, c_{i} \in \mathbb{N}^{*}\right\}$ returns a set of alternative capacities of storage $S$,

$|\xi(s \in S)|= \begin{cases}>1, & \text { if } s \text { has a variable storage capacity } \\ 1, & \text { if } s \text { has not a variable storage capacity }\end{cases}$

- $C_{s} \subseteq(S \times$ Proc $) \cup($ Proc $\times S)$ is the set of connections between memory storage and processors,

- $R \subseteq$ Proc $\cup S$ is the set of resource components (i.e. processors and memory storage),

- $\theta: R \rightarrow \mathbb{B}$ return true if a component (i.e. processor or memory storage) is optional,

- $\phi_{\text {requires }}: R \rightarrow R$ captures dependency between resource components, similarly $\phi_{\text {excludes }}: R \rightarrow R$ captures incompatibility.

The set of, input memories to a processor function $I(f)$, output memories from a processor function $O(f)$ are denoted by:

$\exists p=\left(F, B, C_{b}\right) \in$ Proc,

$I(f \in F):\left\{m \in S \cup B \mid(m, p) \in C_{s} \vee(m, f) \in C_{b}\right\}$,

$O(f \in F):\left\{m \in S \cup B \mid(p, m) \in C_{s} \vee(f, m) \in C_{b}\right\}$.

As a variable platform represents multiple platform configurations, we also capture implicitly all these configurations by introducing several functions, $\theta$ manages the optionality of a resource component. If $\theta(r)=\perp$ the resource is mandatory, otherwise the resource is optional, $\phi_{\text {requires }}$ and $\phi_{\text {excludes }}$ manages constrained relations of dependency and incompatibility between resource components. $\phi_{\text {requires }}(r)=r_{0}$ means that if $r$ is implemented then $r_{0}$ must be implemented too. $r$ depends on $r_{0}$. On the contrary $\phi_{\text {excludes }}(r)=r_{0}$ means that $r$ and $r_{0}$ cannot be implemented on the same platform variant. $r$ and $r_{0}$ are alternatives.

As memory storage can have alternative capacities, we introduce the function $\xi$ which returns the set of alternative capacities $C=$ $\xi(s)$, each memory storage has at least one size and if $|\xi(s)|>1$ the capacity of $s$ is variable.

If the platform has no component variability $\forall r \in R, \theta(r)=\perp$ and no variable memory storage, $\forall s \in S,|\xi(s)|=1$, the platform is not variable.

The platform specification of the case study $V G_{u c}$ is then formalized as

$\left(\operatorname{Proc}_{u c}=\{D C U, G P U\}, S_{u c}=\{R A M, R O M\}\right.$,

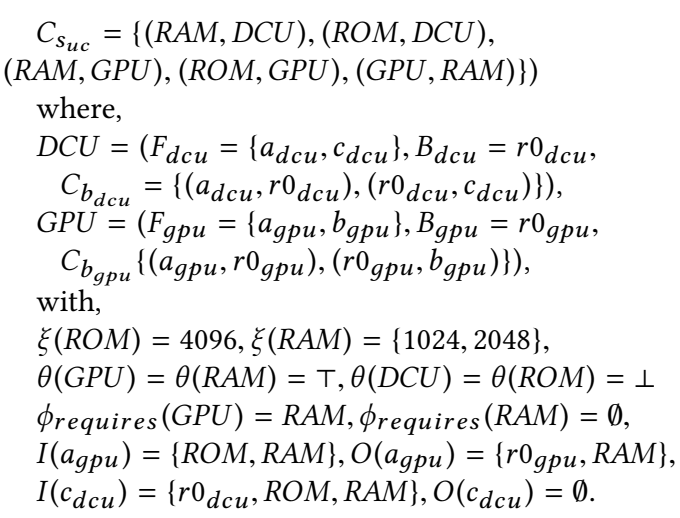

\subsection{Variability-Aware Mapping Process}

The mapping algorithm takes as inputs the variable data-flow and configurable platform models in order to find all embedded system implementations. We propose a mapping model to not only capture all implementations of a single data-flow into a single platform but to capture all data-flow variants implementations onto all platform configurations. Our variability-aware mapping model can be seen as a product line of traditional mapping models.

Definition 3. A variability-aware data-flow-oriented mapping $V M=(T m, D m, E m)$ where:

- $T m \subseteq T \times F$ is the set of possible mappings of tasks on processors $\forall(t, f) \in T m, t$ can be mapped on processor function $f$ because $f$ can implement $t$,

- $D m \subseteq D \times S$ is the set of mappings of data on memory storage,

- $E m \subseteq E \times(S \cup B)$ is the set of data-paths mapping on memory by which data are consumed/produced.

Definition 4. The Variability-Aware Mapping function $M=$ $V D G \times V R G \rightarrow V M$ sorts topologically the data-flow and finds appropriate mapping for each data, task and data-paths of the dataflow using resources of the resource graph.

Basically, each valid mapping should respect some constraints such as that

(1) All tasks are mapped to, a least, one processor function:

$\forall t \in T, \exists(t, f) \in T m$,

(2) All data are mapped to, at least, one memory storage:

$\forall d \in D, \exists(d, s) \in D m$,

(3) All data-paths are mapped to, at least, one appropriate mapping. For data-path starting by an input datum, the storage on which the datum is mapped has to be reachable by the processor function on which the task consuming the datum is mapped.

$\forall e=(d \in D, p, t) \in E, \exists(e, s \in S) \in E m$,

$\exists(d, s) \in D m, \exists(t, f) \in T m, s \in I(f)$,

For data-path between tasks, the memory on which the output of the first task is mapped has to be reachable by the processor function on which the second task is mapped.

$\forall e=\left(t \in T, p, t^{\prime}\right) \in E, \exists(e, m) \in E m$,

$\exists(t, f) \in T m, \exists\left(t^{\prime}, f^{\prime}\right) \in T m, m \in O(f) \wedge m \in I\left(f^{\prime}\right)$

The mapping model of the case study $V M_{u c}$ is then formalized as $\left(T m_{u c}=\left\{\left(a, a_{d c u}\right),\left(a, a_{g p u}\right),\left(b, b_{g p u}\right),\left(c, c_{d c u}\right)\right\}\right.$, $D m_{u c}=\left\{\left(d_{1}, R A M\right),\left(d_{1}, R O M\right),\left(d_{2}, R A M\right),\left(d_{2}, R O M\right)\right\}$, 
$E m_{u c}=\left\{\left(\left(d_{1}, p_{1}, a\right), R A M\right),\left(\left(d_{1}, p_{1}, a\right), R O M\right)\right.$,

$\left(\left(d_{1}, p_{1}, b\right), R A M\right),\left(\left(d_{1}, p_{1}, b\right), R O M\right)$,

$\left(\left(a, p_{2}, c\right), r 0_{d c u}\right),\left(\left(a, p_{2}, c\right), R A M\right),\left(\left(b, p_{2}, c\right), R A M\right)$,

$\left.\left.\left(\left(d_{2}, p_{3}, c\right), R A M\right),\left(\left(d_{2}, p_{3}, R O M\right)\right)\right\}\right)$

Finally, The design space representing all system implementations, called variability-intensive embedded system design space is then composed of a data-flow, platform and mapping:

$V D S \subseteq(V D G \times V R G \times V M)$.

\subsection{Design Space as a Behavioral Product Line}

Automata and model-checking techniques have been widely used to model and validate real-time and embedded systems [4, 5]. Interestingly, the basic approach used is to schedule an application automaton using a platform automaton [11]. Unfortunately, these approaches are not design to manage any variability aspect of specifications.

Our framework relies on Featured-Transition-Systems (FTS) to represent and validate the design space. FTS has the strength to model explicitly the variability points structurally, through a Feature Diagram[3] (FD), instead of modeling variability points behaviorally, by optional transition with possible constraints[24]. This eases the transformation to featured automaton and the removal of invalid implementations from it. In our approach, we also use $L T L$ property to ensure that all valid execution paths of all system implementations reach the end state of all task of the data-flow.

Definition 5. A featured automaton is a tuple $F A=$

(Loc, Loc $0, I$, Act $\subseteq$ Aff $\cup \phi \cup$ Com, trans, $\chi, C h, L, A P, d, \lambda$ ) such that:

- Loc is a finite set of locations, Loc $_{0} \in$ Loc, is a set of initial states and $I \in$ Loc, is a set of final states,

- Ch is a finite set of communication channels,

- $\chi$ is a finite set of variables,

- Act is a set of, Aff which is a finite set of variable affectations, $\phi$ which is a finite set of guards in a boolean expression form and Com $\subseteq\{c ! m, c ? m, c ! ? m \mid c \in C h, m \in \chi\}$, which is a set of communications,

- trans $\subseteq$ Loc $\times$ Act $\times$ Loc are state transitions,

- $d=\left(N \subseteq N_{m} \cup N_{o p t} \cup N_{x o r}, D E \subseteq N \times N, T c l\right)$ is a Feature Diagram (FD), $N$ is the set of mandatory, optional and alternatives features, $D E$ represents relation between features, $T c l$ are constraints between features, $\llbracket d \rrbracket_{F D} \subseteq \mathcal{P}(N)$ is the set of valid product configurations,

- $\lambda:$ trans $\rightarrow \mathbb{B}(N)$ is a total function that labels transitions with feature expressions.

- AP is a set of atomic proposition and L : Loc $\rightarrow$ AP labels transitions with atomic propositions.

A transition $s \stackrel{\alpha}{\longrightarrow} s^{\prime}$ is possible for the set of product configurations $P \subseteq \llbracket \lambda\left(s \stackrel{\alpha}{\rightarrow} s^{\prime}\right) \rrbracket$ and if

$\forall g \in \alpha \cap \phi, g$ is satisfied,

$\forall(c ? m) \in \alpha \cap$ Com, wait for data event $c ! m$,

$\forall\left(c ! ? m_{0}\right) \in \alpha \cap C o m$, send data event $c ! m_{0}$ but wait for data event $c ! m_{1}$ with $m_{0}=m_{1}$.

Definition 6. A Linear Temporal Logic property $(L T L)$ is a temporal expression of atomic proposition that all possible executions of system variants should satisfy as, $\llbracket f a \rrbracket_{F A}=\varphi$ where $\varphi::=a \in A P|\varphi \wedge \varphi| \diamond \varphi$. Symbol $\diamond$ means that the property will become true at some point in the future.

We now show how our design space is transformed to a $F A$. To simplify the transformation process, let us use the following functions:

$$
\begin{aligned}
& f: T \cup \text { Path } \cup R \rightarrow N, f_{s}: D \cup S \times \mathbb{N}^{*} \rightarrow N, \\
& f_{\text {to }}: \text { Path } \rightarrow N, f_{\text {from }}: \text { Path } \rightarrow N, \\
& f_{\text {to }}: \text { Path } \times T \rightarrow N, f_{\text {from }}: T \cup D \times \text { Path } \rightarrow N, \\
& f_{m}: T \cup \text { Path } \rightarrow N, f_{\text {tm }}: T \times F \rightarrow N, f_{\text {pm }}: \text { Path } \times S \cup B \rightarrow N,
\end{aligned}
$$
which transforms model elements to features.

For example, in our case study, the functions would be:

$f(a)=A, f_{s}\left(d_{2}, 1024\right)=D_{2}$ Size 1024

$f_{\text {to }}\left(p_{1}\right)=p_{1} \_$To,$f_{\text {from }}\left(p_{\text {from }}\right)=p_{1 \_ \text {From }}$,

$f_{\text {to }}\left(p_{1}, a\right)=P_{1}$ ToA $f_{\text {from }}\left(a, p_{2}\right)=P_{2}$ From $A$,

$f_{m}(a)=A_{m}, f_{m}\left(p_{1}\right)=P 1_{m}$,

$f_{t m}\left(a, a_{d c u}\right)=A O n A_{d c u}, f_{p m}\left(p_{1}, R O M\right)=p_{1}$ OnROM

$f(R A M)=R A M, f_{s}(R A M, 1024)=$ RAMSize $1024, \ldots$

Similarly,

$c: T \cup D \cup P a t h \cup F \cup S \rightarrow C h$,

$c_{m}: T \cup$ Path $\rightarrow C h$

transforms model elements to communication channels to interact with them at automaton level.

A first function $G_{F A}: V D G \rightarrow F A \times L T L$ transforms a variable data-flow graph into a $F A$ and generates the $L T L$ property in the following way.

(1.1) it transforms each datum $d$ with variable size into a xor feature group (Cf. fig.4(a)): $\zeta(d)>1 \Longrightarrow \forall s \in \zeta(d), \exists\left(f(d) \in N_{x o r}, f_{s}(d, s)\right) \in D E$

(1.2) it creates the automaton for each source datum $d$ (Cf.fig.4(b)), after setting the datum size, calling the mapping automaton (Cf. fig. 6(a,b)) that will allocate the datum on the memory. $\forall s \in \zeta(d), \exists\left\{t_{0}=\left(s_{0} \stackrel{\text { size }(\text { in })=s}{\longrightarrow} s_{1}\right)\right.$, where,
$\quad|\zeta(d)|>1 \Longrightarrow \lambda\left(t_{0}\right)=f_{s}(d, s)$,
$\quad \forall p \in O(d), c_{m}(p) ! ?$ in
$\left.s_{1} \stackrel{\forall}{\longrightarrow} s_{2}, s_{2} \stackrel{\forall p \in O(d), c(p) ! \text { in }}{\longrightarrow} s_{3}\right\} \in$ trans

(2.1) it transforms each variable data-path $p$ in a xor feature group (Cf. fig. 4(a)).

$|O(p)|>1 \Longrightarrow \forall o \in O(p)$,

$\exists\left(f_{\text {to }}(p) \in N_{\text {xor }}, f_{\text {to }}(p, o)\right) \in D E$

$|I(p)|>1 \Longrightarrow \forall i \in I(p)$,

$\exists\left(f_{\text {from }}(p) \in N_{\text {xor }}, f_{\text {from }}(i, p)\right) \in D E$

(3.1) it creates task/data-paths consistency constraints (Cf. fig. $4(\mathrm{a}))$.

$$
\begin{aligned}
& \forall t \in T, \forall p \in|I(t)|,|O(p)|>1 \Longrightarrow \\
& \quad \exists\left(f(t) \Longleftrightarrow f_{t o}(p, t)\right) \in T c l \\
& \forall t \in T, \forall p \in|O(t)|,|I(p)|>1, \\
& \quad \exists\left(f(t) \Longleftrightarrow f_{\text {from }}(t, p)\right) \in T c l
\end{aligned}
$$

(3.2) it creates for each task $t$ the automaton (Cf. fig. 4(c)) that will wait for data-paths allocation, then call the mapping automaton (Cf. fig. 6(c)) to execute the task.

$$
\begin{aligned}
& \exists\left\{t_{0}=\left(s_{0} \stackrel{\forall p \in I(t), c(p) ? \text { in }}{\longrightarrow} s_{1}\right),\right. \text { where, } \\
& \quad f(t) \in N_{\text {opt }} \Longrightarrow \\
& \quad \lambda\left(t_{0}\right)=f(t) \wedge \lambda\left(\left(s_{0} \rightarrow s_{4}\right) \in \text { trans }\right)=\neg f(t), \\
& s_{1} \stackrel{\forall p \in O(t), c_{m}(p) ! ? \text { out }}{\longrightarrow} s_{2}, s_{2} \stackrel{c_{m}(t) ! ? \text { in }, \text { out }}{\longrightarrow} s_{3},
\end{aligned}
$$


$s_{3} \stackrel{\forall p \in O(t), c(p) ! \text { out }}{\longrightarrow} s_{4} \in I$,

where, $\left.L\left(s_{4}\right)=t_{\text {end }} \in A P\right\} \in$ trans

(3.3) it generates the $L T L$ formula that checks that a valid execution must, at some point, satisfy atomic proposition of all data-flow task terminal states.

$\varphi=\diamond\left(\wedge_{s \in I, L(s) \neq \emptyset} L(s)\right)$

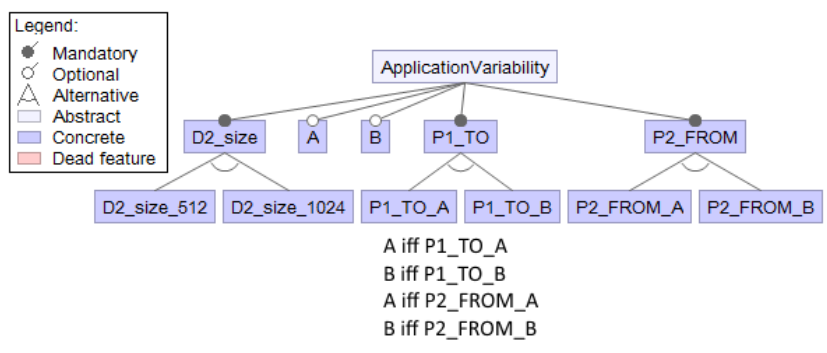

(a) Application FD

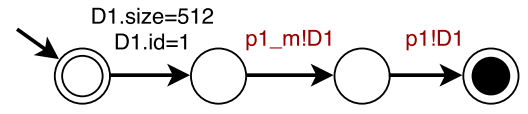

(b) Datum $d_{1}$ FA

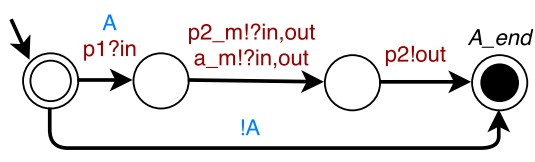

(c) Task A FA

Figure 4: Partial variable data-flow application FA

The second function $G_{F A}: V R G \rightarrow F A$ transforms a variable resource graph into a $F A$ in the following way.

(1) it creates feature constraints on resource implementation (Cf. fig. 5(a)).

$\forall r \in R, \theta(r)=\top \Longrightarrow \exists f(r) \in N_{o p t}$

$\forall r \in R, \forall r_{\text {req }} \in \phi_{\text {requires }}(r), \exists\left(f(r) \Longrightarrow f\left(r_{r e q}\right)\right) \in T c l$

$\forall r \in R, \forall r_{\text {exc }} \in \phi_{\text {excludes }}(r), \exists\left(f(r) \Longrightarrow \neg f\left(r_{\text {exc }}\right)\right) \in$ $\mathrm{Tcl}$

(2.1) it creates for each storage $s$ features representing storage alternative sizes.

$\forall c \in \xi(s), \exists\left(f(s) \in N_{\text {xor }}, f_{s}(s, c)\right) \in D E$

(2.2) it creates for each storage $s$ an automaton that represents basic memory behavior (Cf. fig. 5(b)), cons and cap are respectively the consumed size and the maximal capacity of the storage. Through channels, one can allocate memory, and if there is not enough memory, an error is raised.

$\forall c \in \xi(s), \exists\left\{t_{0}=\left(s_{0} \stackrel{\text { cons }=0}{\longrightarrow} s_{1}\right)\right.$, where,

$f(s) \in N_{\text {opt }} \Longrightarrow$

$\lambda\left(t_{0}\right)=f(s) \wedge \lambda\left(\left(s_{0} \rightarrow s_{4}\right) \in\right.$ trans $)=\neg f(s)$,

$s_{1} \stackrel{c a p=c}{\longrightarrow} s_{2}$, where, $\lambda\left(s_{1} \stackrel{c a p=c}{\longrightarrow} s_{2}\right)=f(s, c)$,

$s_{2} \stackrel{c(s) ? \text { in, cons }+=\text { size }(\text { in })}{\longrightarrow} s_{3}, s_{3} \stackrel{\text { cons }<\text { size }}{\longrightarrow} s_{2}$,
(3) it creates for each processor $p$ an automaton that models basic graphic processor pipeline behavior (cf. fig. 5(c)). When a processor function is executed, the input and output are checked to verify that the pipeline is not misused.

$\forall p=\left(F, B, C_{b}\right) \in$ Proc,$\forall f \in F$,

$\forall\left(s_{i}, p\right) \in C_{s}, \forall\left(p, s_{o}\right) \in W_{s}, \forall\left(b_{i}, f\right) \in C_{b}, \forall\left(f, b_{o}\right) \in C_{b}$, $\exists\left\{t_{0}=\left(s_{0} \stackrel{c(f) \text { ?in, out }}{\longrightarrow} s_{1}\right)\right.$, where,

$f(p) \in N_{o p t} \Longrightarrow$

$\lambda\left(t_{0}\right)=f(p) \wedge \lambda\left(\left(s_{0} \rightarrow s_{4}\right) \in\right.$ trans $)=\neg f(p)$,

$s_{1} \stackrel{\operatorname{loc}(\text { in })=s_{i} \wedge \forall\left(b_{i}, f\right) \in R_{b}, b_{i}=\text { free }}{\longrightarrow} s_{2}$,

$s_{1} \stackrel{\operatorname{loc}(\text { in })=b_{i} \wedge b_{i}=i n}{\longrightarrow} s_{2}$,

$s_{2} \stackrel{\text { loc }(\text { out })=s_{o} \wedge \forall\left(f, b_{o}\right) \in W_{b}, b_{o}=f r e e}{\longrightarrow} s_{3}$,

$\left.s_{2} \stackrel{\text { loc }(\text { out })=b_{o} \wedge b_{o}=\text { free }}{\longrightarrow} s_{3}, s_{3} \stackrel{c(f) ! \text { in }, \text { out }}{\longrightarrow} s_{0}\right\} \in$ trans

A third function $G_{F A}: V M \rightarrow F A$ transforms a variabilityaware dataflow-oriented mapping into a $F A$ as follows.

(1.1) it creates features representing all possible task mappings on processor function (cf. fig. 6(a)).

$\forall(t, f) \in T m, \exists\left(f_{m}(t) \in N_{x o r}, f_{t m}(t, f)\right) \in D E$

(1.2) it creates for each task mapping the automaton that executes the processor function according to the mapping configuration (cf. fig. 6(c)).

$$
\begin{aligned}
& \forall t \in T, \forall(t, f) \in T m, \exists\left\{t_{0}=\left(s_{0} \stackrel{c_{m}(t) \text { ?in, out }}{\longrightarrow} s_{1}\right),\right. \text { where, } \\
& \quad f_{m}(t) \in N_{\text {opt }} \Longrightarrow \\
& \quad \lambda\left(t_{0}\right)=f_{m}(t) \wedge \lambda\left(\left(s_{0} \rightarrow s_{3}\right) \in \text { trans }\right)=\neg f_{m}(t) \\
& t_{1}=\left(s_{1} \stackrel{c(f) ! ? \text { in }, \text { out }}{\longrightarrow} s_{2}\right), \text { where, } \lambda\left(t_{1}\right)=f_{\text {tm }}(t, f), \\
& \left.s_{2} \stackrel{c_{m}(t) ! \text { in,out }}{\longrightarrow} s_{3}\right\}, \in \text { trans }
\end{aligned}
$$

(2.1) Like 1.1, it creates features representing all possible data-path mappings on memory.

$\forall((x, p, y), s) \in E m, \exists\left(f_{m}(p) \in N_{x o r}, f_{p m}(p, s)\right) \in D E$

(2.2) Like 2.2, it creates for each data-path mapping the automaton that allocates memory (Cf. fig.6(b)).

$\forall p \in$ Path,$\forall((x, p, y), s) \in E m$,

$\exists\left\{s_{0} \stackrel{c_{m}(p) \text { ?out }}{\longrightarrow} s_{1}, t_{0}=\left(s_{1} \stackrel{c(s) ! ? o u t, \operatorname{loc}(d)=s}{\longrightarrow} s_{2}\right)\right.$, where,

$\left.\lambda\left(t_{0}\right)=f_{p m}(p, s), s_{2} \stackrel{c_{m}(p) ! \text { out }}{\longrightarrow} s_{3}\right\} \in$ trans

Finally the function $\operatorname{Gen}_{F A}: V D S \rightarrow F A$, defined by:

$\operatorname{Gen}_{F A}((v d g, v r g, v m))$ :

$\operatorname{Gen}_{F A}(v d g)\left\|G e n_{F A}(v r g)\right\| G e n_{F A}(v m)$, transforms our design space into a featured automaton.

To preserve the consistency of the design space, variability constraints are inferred such as:

(1.1) Task features with variable data-path features are not implemented on all data-flow variants, then those features are made optional (Cf. fig. 4(a)).

$\forall t \in T$,

$$
\begin{aligned}
& \exists p_{i} \in I(t),\left|O\left(p_{i}\right)\right|>1 \vee \exists p_{o} \in O(t),\left|I\left(p_{o}\right)\right|>1 \\
& \quad \Longrightarrow f(t) \in N_{o p t}
\end{aligned}
$$

(1.2) Variable task features have their mapping variable too; if a task feature is implemented its mapping must be implemented too, and vice-versa (Cf. fig. 4(a) \& 6(a)).

$\forall t \in T, f(t) \in N_{o p t} \Longrightarrow$ $f_{m}(t) \in N_{\text {opt }} \wedge\left(f(t) \Longleftrightarrow f_{m}(t)\right) \in T c l$ 


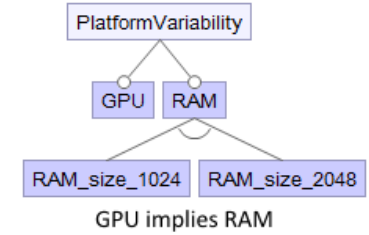

(a) Platform FD

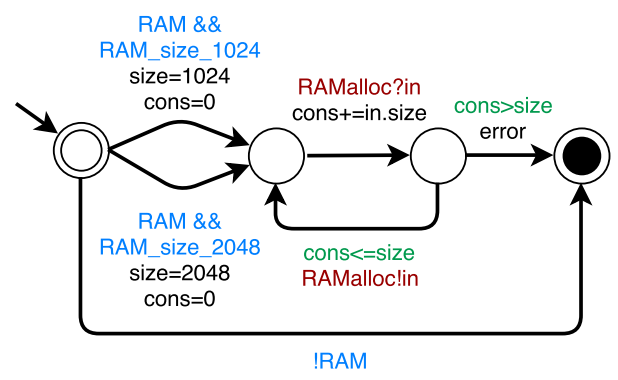

(b) RAM storage

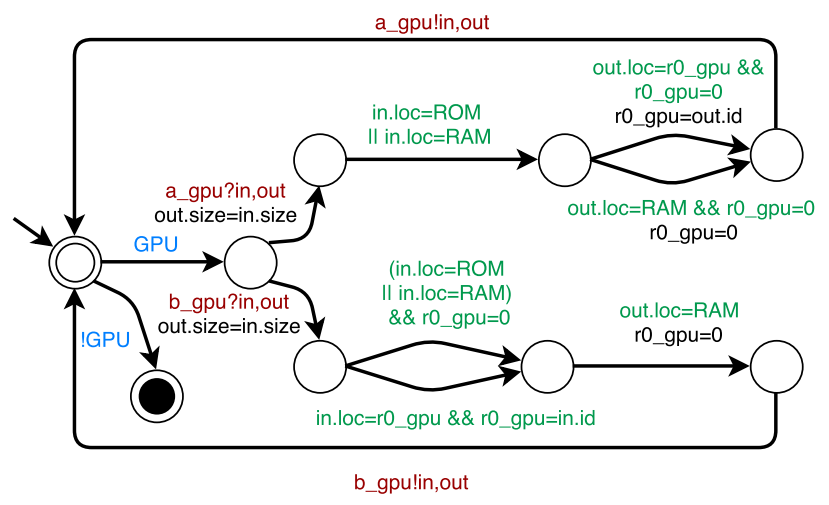

(c) GPU processor

Figure 5: Partial variable platform FA

(2.1) If a task mapping feature is implemented on a processor function, the implemented input and output path mappings have to be reachable (Cf. fig. 6(a)).

$\forall(t, f) \in T m, \forall p_{i} \in I(t), \forall p_{o} \in O(t)$,

$$
\begin{aligned}
& \exists\left(f_{t m}(t, f) \underset{\quad \Longleftrightarrow}{(} \underset{\left(\left(x, p_{i}, t\right), m \in I(f)\right) \in E m}{\bigvee} f_{p m}\left(p_{i}, m\right)\right) \wedge \\
& \quad\left(\left(t, p_{o}, x\right), m^{\prime} \in O(f)\right) \in E m \\
& \left.\left.f_{p m}\left(p_{o}, m^{\prime}\right)\right)\right) \in T c l
\end{aligned}
$$

(3.1) If a task mapping feature using an optional processor is implemented, the processor must be implemented too. $\forall p=(F, x, y) \in$ Proc, $f(p) \in N_{o p t}, \forall(t, f \in F) \in T m$

$$
\Longrightarrow \exists\left(f_{t m}(t, f) \Longrightarrow f(p)\right) \in T c l
$$

Similarly, if a data-path mapping feature is implemented on fifo buffer of optional processor (3.2) or optional memory storage (3.3), the resource have to be implemented.

(3.2) $\forall p u=(F, B, x) \in \operatorname{Proc}, \forall((y, p, z), b \in B) \in E m$,

$$
f(p u) \in N_{o p t} \Longrightarrow \exists\left(f_{p m}(p, b) \Longrightarrow f(p u)\right) \in T c l
$$

(3.3) $\forall((x, p, y), s \in S) \in E m, f(s) \in N_{o p t} \Longrightarrow$ $\exists\left(f_{p m}(p, s) \Longrightarrow f(s)\right) \in T c l$

As an illustration, in our case study, the rules would be:

(1.2) $A \Longleftrightarrow A_{m}, B \Longleftrightarrow B_{m}$

(3.1) $B O n B_{g p u} \Longrightarrow G P U, A O n A_{g p u} \Longrightarrow G P U$

(3.2) $\mathrm{P} 2 \mathrm{OnR}_{\mathrm{gpu}} \Longrightarrow \mathrm{GPU}$

(3.3) P1OnRAM $\Longrightarrow$ RAM, P2OnRAM $\Longrightarrow$ RAM

P3OnRam $\Longrightarrow R A M$

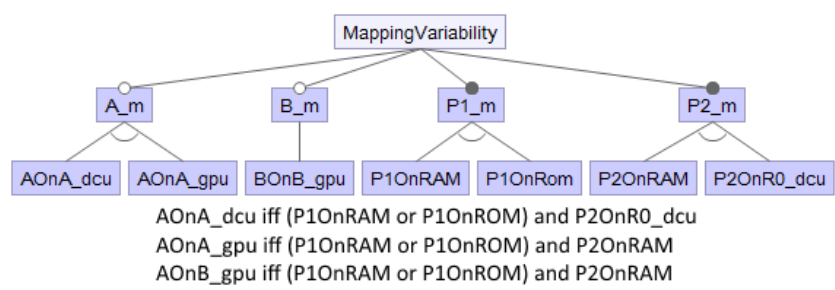

(a) Mapping FD

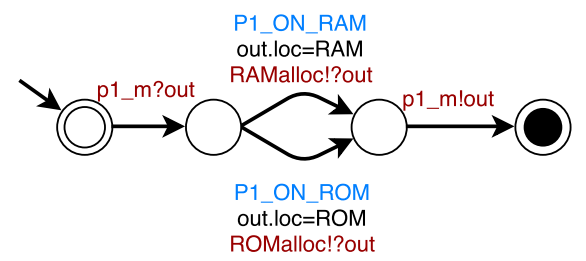

(b) data-path Mapping $p_{1}$ FA

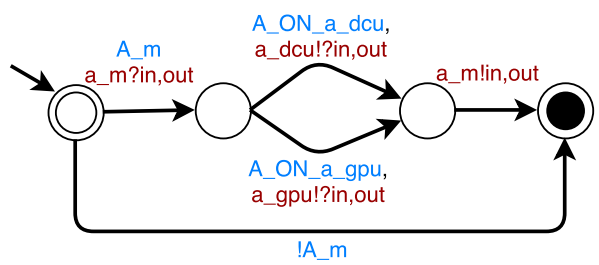

(c) Task Mapping A FA

Figure 6: Partial Variability-Aware Mapping FA

\subsection{Validation Process}

As our form of behavioral product lines is based on FTS [9], model checking techniques can be directly reused. In our implementation (Cf. next section), we reuse the ProVeLines/SNIP checker as a backend for the validation process. The process consists in verifying all execution paths of all products $\llbracket f a \rrbracket_{F A}$ of the product line, in an efficient way by exploiting commonalities between different products. Theoretically, the more the products share common behavior and the more efficient should be the variability aware model checking in comparison of iterative model checking on individual systems [8]. Instead of exploring all executions for each system implementation, the model-checker explores an execution $\pi$ once for all implementations $P$ able to produce this specific execution:

$$
P=\left\{p \in \llbracket d \rrbracket_{F D} \mid \pi \in \llbracket f a_{\mid p} \rrbracket_{A}\right\} .
$$


As mentioned in the previous section, some system configurations may expose inconsistent behaviors (e.g., memory allocation error, violation of graphical pipeline constraints). These behaviors will abort the execution and the basic properties (e.g. safety, absence of deadlock, state reachability) will obviously not be satisfied. In our validation process, we are able to remove these configurations from the system by relying again on the back-end model checker [8]. It computes the set of bad product configurations, which we remove from the feature diagram of the product line by adding the appropriate cross-tree constraints.

\section{EVALUATION}

\subsection{Implementation}

The presented framework has been entirely implemented. The variable application and platform input meta-models, the mapping algorithm and the removal of invalid variants from the variable design space are implemented in Java. Functional and platform variability specifications are entered through a dedicated API, and the framework then generates explicit variability models. The framework implementation also infers knowledge about task implementation capabilities of processors, and generates task, data-path, processor and memory automata by automatically configuring predefined templates.

The mapping algorithm implementation is traversing all model representations to find the appropriate mappings by name matching. Then it generates constrained Feature Models and Featured Automata in TVL [6] and fPromela specifications. Automated reasoning techniques can be used over TVL Feature Models (e.g. SAT solving [17]) to reason about the structural variability of the represented system. As for the validation process of the behavioral product line, it uses the ProVeLines/SNIP model-checker [7, 10], which takes TVL and fPromela files as input.

\subsection{Industrial Use Case}

In order to validate our tooled approach on an industrial scale, we applied it to a real low-end market instrument cluster provided by Visteon, the automotive systems company we collaborate with.

The functional requirements of the cluster represents a variable data-flow with 3 source images processed by 8 tasks connected by 9 data-paths. Each source image has two different resolutions (i.e HD and LD) and two tasks sub flow sequences are alternative through a xor join/split data-path, resulting in 16 data-flow variants. The platform specification of the cluster is then represented by a variable hardware component system with 2 memories (a Video RAM and a ROM Flash) and 3 processors (two multi-pass GPU bitblitter and one streaming- based DCU). Each processor has a pipeline processing of 4 stages and 3 fifo buffers. In terms of platform variability, the 2 bitblitters and the VRAM are optional. Each memory has 2 different configurable sizes at manufacturing time. The number of platform configurations in the use case is then 24 .

If each data-flow variant had one possible implementation on each platform configuration, the number of different cluster system implementations would be 384 . In reality, some platform configurations do not provide the graphical functionalities required by some data-flow variants. Furthermore, due to multiple task implementation choices, data-flow variants have several thousand possible implementation alternatives onto a platform configuration. Setting the platform configuration to the higher end (i.e. selecting VRAM and all processors), one can find 72 and 78 possible implementations for two data-flow variants that take different xor data-path decisions. This is due to more pipelining opportunities in the second data-flow variant, even if there is more data-path mapping possibilities in the first one.

Table 1 shows time measurements of the complete toolchain while varying the different variability dimensions over the use case. In the first seven rows, we observe that the whole process is performing well with small to medium scale of variabilities. Data and memory size variability verifications are faster and require more state exploration than platform component and data-path variabilities. Component and data-path variabilities are also slower to check than data and memory size variabilities. It is likely to be due to the fact that contrary to size variability, hardware component and data-path variability are strongly impacting the implementation variability, and consequently the state space of the model checker.

We also have complemented this experimentation by taking a single structural data-flow from the industrial use case with a simulated larger platforms with multiple memories and processors. Results in the last three rows of Table 1 show that the solving can scale to a large number of implementation variants, even if the solving time is significant.

\section{CONCLUSION}

A tremendous amount of variability can be observed in embedded systems, and especially in data-flow oriented ones, which are now systematically built from highly variable specifications and target diverse hardware platforms configurable at a very high level of detail. To handle the early functional assessment of all these possible configurations, we proposed in this paper a tooled framework that takes variable data-flow specifications and variable hardware platform models to map them together and transform them into a behavioral product line representing the potential design space. The framework allows to use automated reasoning techniques to explore and assess the feasibility of all represented variants in a single run, and invalid products can be removed by adding constraints to the product line. We also reported on the application of the proposed approach to a real-world industrial use case of automotive instrument cluster, showing its potential good applicability.

As future work, we first plan to facilitate the usage of the framework with domain specific languages for input models (specification and platform), and to conduct larger experiments with them. We will also extend the framework by providing guidance when conducting functional validation, and by taking into account nonfunctional properties (e.g. cost) so to provide optimized product selection as a complement to the functional validation presented in this paper.

\section{ACKNOWLEDGMENTS}

We thank Visteon Electronics and ANRT for continuously supporting this research, especially, Emmanuel Roncoroni and Olivier Bantiche who brought industrial knowledge and expertise in instrument cluster engineering. 


\begin{tabular}{|c|c|c|c|c|c|c|c|}
\hline Variability & $\begin{array}{c}\text { Implementation } \\
\text { variants }\end{array}$ & $\begin{array}{c}\text { Platform } \\
\text { variants }\end{array}$ & $\begin{array}{c}\text { data-flow } \\
\text { variants }\end{array}$ & $\begin{array}{c}\text { States explored } \\
\text { (re-explored) }\end{array}$ & $\begin{array}{c}\text { Time } \\
(\mathrm{ms})\end{array}$ & $\begin{array}{c}\text { ms / } \\
\text { variants }\end{array}$ & $\begin{array}{c}\text { states / } \\
\text { variants }\end{array}$ \\
\hline \hline NONE & 78 & 0 & 0 & $2453(331)$ & 27 & 0.346 & 31.448 \\
\hline Data size & 624 & 0 & 8 & $15254(2406)$ & 201 & 0.322 & 35.976 \\
\hline Platform & 424 & 24 & 0 & $5673(546)$ & 65 & 0.153 & 13.379 \\
\hline Platform and data size & 3392 & 24 & 8 & $37435(4856)$ & 602 & 0.177 & 11.036 \\
\hline data-path & 150 & 0 & 2 & $4727(981)$ & 74 & 0.493 & 31.513 \\
\hline data-path and data size & 1200 & 0 & 16 & $29066(6994)$ & 587 & 0,489 & 24,222 \\
\hline ALL & $>4800$ & 24 & 16 & $72704(14652)$ & 2361 & - & - \\
\hline \hline Platform mult. mem & 16408 & 40 & 0 & $134941(4534)$ & 4010 & 0.244 & 8.224 \\
\hline Platform mult. proc & 2848 & 80 & 0 & $19625(3224)$ & 337 & 0.118 & 6.890 \\
\hline Pltf. mult. proc \& mem & 516608 & 160 & 0 & $1341999(175304)$ & 289721 & 0.560 & 2.597 \\
\hline
\end{tabular}

Table 1: Industrial use case results

\section{REFERENCES}

[1] Patrizia Asirelli, Maurice H ter Beek, Stefania Gnesi, and Alessandro Fantechi. 2011. Formal description of variability in product families. In Software Product Line Conference (SPLC), 2011 15th International. IEEE, 130-139.

[2] Felice Balarin. 1997. Hardware-software co-design of embedded systems: the POLIS approach. Springer Science \& Business Media.

[3] Don Batory. 2005. Feature models, grammars, and propositional formulas. In SPLC, Vol. 3714. Springer, 7-20.

[4] Johan Bengtsson, Kim Larsen, Fredrik Larsson, Paul Pettersson, and Wang Yi. 1996. UPPAAL-a tool suite for automatic verification of real-time systems. Hybrid Systems III (1996), 232-243.

[5] Edmund M Clarke, Orna Grumberg, and Doron Peled. 1999. Model checking. MIT press.

[6] Andreas Classen, Quentin Boucher, and Patrick Heymans. 2011. A text-based approach to feature modelling: Syntax and semantics of TVL. Science of Computer Programming 76, 12 (2011), 1130-1143.

[7] Andreas Classen, Maxime Cordy, Patrick Heymans, Axel Legay, and Pierre-Yves Schobbens. 2012. Model checking software product lines with SNIP. Int. fournal on Software Tools for Technology Transfer (STTT) (2012), 1-24.

[8] Andreas Classen, Patrick Heymans, Pierre-Yves Schobbens, and Axel Legay. 2011 Symbolic model checking of software product lines. In Proceedings of the 33rd International Conference on Software Engineering. ACM, 321-330.

[9] Andreas Classen, Patrick Heymans, Pierre-Yves Schobbens, Axel Legay, and Jean-François Raskin. 2010. Model checking lots of systems: efficient verification of temporal properties in software product lines. In Proceedings of the 32nd ACM/IEEE International Conference on Software Engineering-Volume 1. ACM, 335344 .

[10] Maxime Cordy, Andreas Classen, Patrick Heymans, Pierre-Yves Schobbens, and Axel Legay. 2013. ProVeLines: a product line of verifiers for software product lines. In Proceedings of the 17th International Software Product Line Conference co-located workshops. ACM, 141-146.

[11] Andreas E Dalsgaard, Mads Chr Olesen, Martin Toft, René Rydhof Hansen, and Kim Guldstrand Larsen. 2010. Metamoc: Modular execution time analysis using model checking. In OASIcs-OpenAccess Series in Informatics, Vol. 15. Schloss Dagstuhl-Leibniz-Zentrum fuer Informatik.

[12] Sebastian Graf, Michael Glaß, Jurgen Teich, and Christoph Lauer. 2014. Multivariant-based design space exploration for automotive embedded systems. In Design, Automation and Test in Europe Conference and Exhibition (DATE), 2014 IEEE, $1-6$.

[13] Sebastian Graf, Michael Glaß, Dominic Wintermann, Jurgen Teich, and Christoph Lauer. 2013. IVaM: Implicit variant modeling and management for automotive embedded systems. In Hardware/Software Codesign and System Synthesis (CODES+ ISSS), 2013 International Conference on. IEEE, 1-10.

[14] Sebastian Graf, Sebastian Reinhart, Michael Glaß, Jurgen Teich, and Daniel Platte. 2015. Robust design of E/E architecture component platforms. In 52nd Design
Automation Conference (DAC). IEEE, 1-6.

[15] Matthias Gries. 2004. Methods for evaluating and covering the design space during early design development. Integration, the VLSI journal 38, 2 (2004), 131-183.

[16] Bart Kienhuis, Ed Deprettere, Kees Vissers, and Pieter Van Der Wolf. 1997. An approach for quantitative analysis of application-specific dataflow architectures. In Application-Specific Systems, Architectures and Processors, 1997. Proceedings., IEEE International Conference on. IEEE, 338-349.

[17] Marcilio Mendonca, Andrzej Wasowski, and Krzysztof Czarnecki. 2009. SATbased analysis of feature models is easy. In Proceedings of the 13th International Software Product Line Conference. Carnegie Mellon University, 231-240.

[18] Gianluca Palermo, Cristina Silvano, and Vittorio Zaccaria. 2008. Robust optimization of SoC architectures: A multi-scenario approach. In Embedded Systems for Real-Time Multimedia, 2008. ESTImedia 2008. IEEE/ACM/IFIP Workshop on. IEEE, $7-12$.

[19] Gianluca Palermo, Cristina Silvano, and Vittorio Zaccaria. 2009. Variability-aware robust design space exploration of chip multiprocessor architectures. In Design Automation Conference, ASP-DAC. Asia and South Pacific. IEEE, 323-328.

[20] Lars Schor, Iuliana Bacivarov, Devendra Rai, Hoeseok Yang, Shin-Haeng Kang, and Lothar Thiele. 2012. Scenario-based design flow for mapping streaming applications onto on-chip many-core systems. In Proceedings of the 2012 international conference on Compilers, architectures and synthesis for embedded systems. ACM, $71-80$

[21] Kamana Sigdel, Mark Thompson, Andy D Pimentel, Carlo Galuzzi, and Koen Bertels. 2009. System-level runtime mapping exploration of reconfigurable architectures. In Parallel \& Distributed Processing, 2009. IPDPS 2009. IEEE International Symposium on. IEEE, 1-8.

[22] Vlad-Mihai Sima and Koen Bertels. 2009. Runtime decision of hardware or software execution on a heterogeneous reconfigurable platform. In Parallel \& Distributed Processing, 2009. IPDPS 2009. IEEE International Symposium on. IEEE, $1-6$

[23] Amit Kumar Singh, Muhammad Shafique, Akash Kumar, and Jörg Henkel. 2013. Mapping on multi/many-core systems: survey of current and emerging trends. In Proceedings of the 50th Annual Design Automation Conference. ACM, 1.

[24] Maurice H ter Beek, Alessandro Fantechi, Stefania Gnesi, and Franco Mazzanti. 2016. Modelling and analysing variability in product families: model checking of modal transition systems with variability constraints. Fournal of Logical and Algebraic Methods in Programming 85, 2 (2016), 287-315.

[25] Peter Van Stralen and Andy Pimentel. 2010. Scenario-based design space exploration of MPSoCs. In Computer Design (ICCD), 2010 IEEE International Conference on. IEEE, 305-312.

[26] Stefan Wildermann, Felix Reimann, Jürgen Teich, and Zoran Salcic. 2011. Operational mode exploration for reconfigurable systems with multiple applications. In Field-Programmable Technology (FPT), 2011 International Conference on. IEEE, $1-8$. 